\title{
Management of people with schizophrenia in primary care
}

\author{
Tony Kendrick
}

In 1961 Parkes and his colleagues followed 100 people with schizophrenia for a year after their discharge from hospitals in south London and found that the general practitioner (GP) was the professional most frequently in contact with people. Only $56 \%$ attended as psychiatric outpatients, most being seen fewer than five times in the year, while $70 \%$ were in regular contact with their GPs, and when problems developed it was usually the GP who was involved first (Parkeset al, 1962).

Thirty years later, Melzer et al (1991) carried out a very similar study, also following 100 people with schizophrenia for a year after discharge from hospital in south London. This showed little change in the pattern of care, despite the development of community mental health teams (CMHTs) in the intervening decades. Between nine and 12 months after discharge, $52 \%$ of people attended a psychiatric clinic while $57 \%$ saw their GP. Only $20 \%$ were in contact with community psychiatric nurses (CPNs).

Although the UK Government would like psychiatric teams to be responsible for the care of all people with psychotic illnesses in the community (Department of Health, 1994), surveys have shown consistently that in reality a significant proportion (around 25-30\%) lose contact with psychiatric services and are looked after entirely in general practice (King, 1992). Even where people are in contact with specialist services, they may still need their GP's help too, if only for prescriptions and sick notes. A significant number of people with chronic psychosis receive their regular depot neuroleptic injections from general practice nurses rather than CPNs (details available from the author upon request). It is therefore very important that GPs, practice nurses and other members of the primary care team have an understanding of the problems of people with schizophrenia, and know where to seek help when necessary (see Box 1).

A number of factors recommend the involvement of GPs in the care of people with schizophrenia. They have often known the person as a child before the illness developed, and many people who will not attend psychiatric clinics are still happy to go to their own doctor's surgery for care. They are the family's doctor too - most CMHTs do not offer 24hour care and the GP is usually the first port of call for relatives when problems develop out of hours. Even if the primary care team is not directly involved with the care of the mental health problem, people with schizophrenia have increased needs for physical and preventive health care

Box 1. Need to improve care in general practice

Between 25 and $30 \%$ of people with schizophrenia lose contact with psychiatric services and depend on general practice for their care

Most general practitioners have not had any postgraduate experience in psychiatry

Significant numbers of patients with psychosis receive their depot neuroleptic injections from general practice nurses who have little or no mental health training

General practitioners do not review chronic mental illnesses as often as they do chronic physical illnesses, and individuals can go for years with no review of their care

Tony Kendrick spends half his time working as a GP and the other half as a Reader in General Practice and Primary Care (St George's Hospital Medical School, Hunter Wing, Cranmer Terrace, London SW17 0RE). He was Mental Health Foundation Research Fellow for four years from 1990 to 1994, during which time most of the research quoted was carried out. 
(Allebeck, 1989; Kendrick, 1996), which will usually involve the GP and the practice nurse.

However, there are a number of problems which need to be overcome if good care is to be provided in general practice.

\section{Obstacles to management of schizophrenia in primary care}

\section{Lack of training and experience}

Most GPs have had little psychiatric training, usually eight weeks or so as a medical student, often many years ago. Vocational training has improved the situation, but even among newly trained principals in the 1990s, only $40 \%$ have completed a postgraduate psychiatry senior house job (Styles, 1991). Each GP will have on their books, on average, around seven people with chronic schizophrenia (Jablensky, 1986), which means that they do not have frequent opportunities to learn about the condition through experience with their own patients. Therefore, GPs need up-to-date information and practical advice on the management of schizophrenia. The same is true of practice nurses, the large majority of whom are registered general nurses with little or no mental health training. Mental health courses for practice nurses are almost exclusively directed towards counselling for anxiety and minor depression.

\section{Reactive nature of general practice}

General practice is essentially reactive - GPs wait for people to come and see them or to ask for a home visit. This may be sufficient for those people with schizophrenia who are in remission, or are stable on treatment and have insight into the need to present themselves for regular treatment and review. However, a significant minority of people with schizophrenia do not see their GP from one year to the next and need to be sought out for review. If a person is reluctant to be reviewed and active intervention is needed, referral back to the CMHT is indicated - with 2000 people on the average patient list, GPs have little time to spend actively seeking people. However, GPs and practice nurses need to be aware of people who do not attend for medication and review, in order to alert the CMHT to find out whether individuals are running into problems. This involves reversing the usual assumption in primary care, which is that if a person does not return, it means they are well. To be aware of who is and is not attending for care, the practice ideally needs a register of people with schizophrenia.

\section{Limited time for assessments}

The third problem is that contacts in primary care are relatively short. On average, a person spends eight minutes in the GP's surgery, perhaps longer on home visits. This is the nature of general practice, where 40 or more people need attention from the doctor every day. Practice nurse appointments may be only five minutes long for a depot injection. Therefore, GPs and practice nurses need a strategy to identify important problems or changes in people with schizophrenia quickly and efficiently. They simply do not have the time to carry out a lengthy comprehensive psychiatric and social assessment of the kind that might be performed in specialist out-patient clinics.

Traditionally, GPs have been taught that the most efficient consulting style is to start with an open question and encourage the person to set the agenda. This ensures that the person's concerns are quickly identified and can be addressed in the time available. However, this approach may be inappropriate for many people with schizophrenia. Those with negative symptoms including apathy, inactivity, a poor self-image, self-neglect and indifference to discomfort, are unlikely to bring problems to their GP's attention. A number of problems can build up, which could lead to a crisis in care if not detected. Therefore, a repertoire of specific closed questions are needed to ensure that problems are not missed.

\section{General practitioners' perceptions of their role}

We carried out a survey of 507 GPs in South Thames West Region in 1990, asking them about their involvement with people with disabling long-term mental disorders like schizophrenia (Kendrick et $a l, 1991)$. The majority of the 369 (73\%) who responded estimated that they had fewer than 10 such persons each, although those practising in London or within three miles of a large mental hospital had more than average. Most agreed that some long-term mentally disordered people came to their attention only when there was a crisis, and that communication was often a problem, yet only nine GPs had specific practice policies for their care. Most GPs were willing to share the care of such 
people with a psychiatrist, with the CPN as the person's keyworker.

So how can primary care teams, together with specialists, improve the care they offer to people with schizophrenia?

\section{Setting up case registers}

Studies have shown that the large majority of people with schizophrenia and other severe mental disorders on the GP's list can be quickly identified, especially now that most practices are computerised. Compared to a laborious trawl through all the practice's written case notes, the sensitivity and specificity of diagnostic labels of schizophrenia on computer were found to be greater than $90 \%$, in London practices using the desk-top Vamp system. Over $90 \%$ of prescriptions for antipsychotic drugs were recorded on the practices' computers ( Nazareth et al, 1993a).

We carried out a study at St George's Hospital Medical School of the care of people with disabling long-term mental disorders in 16 practices in South Thames Region (Kendrick et al, 1994). Over $90 \%$ of people were quickly identified by listing all those already known to the primary care team, combined with searching repeat prescription systems for psychotropic drugs, and asking the local psychiatrist, CPN, social services case manager and day hospital staff for the names of any people known to them who were registered with the practices. It should not be difficult for most practices to identify people with schizophrenia quickly using these methods.

Having identified the people, the practice should check their records for current contact with psychiatric services - the GP should be informed of all persons in receipt of the Care Programme Approach (Department of Health, 1994). People who are out of contact may benefit from referral back to secondary care, although a proportion will decline referral, preferring to see their GP for continuing care.

\section{Making the most of patients' contacts with their GPs}

In our study, entries in the case notes of 204 people with schizophrenia found in the 16 practices showed that within the previous 12 months $57 \%$ had seen a psychiatrist, $43 \%$ a CPN, $71 \%$ had seen either a psychiatrist or a CPN, and $7 \%$ had seen a social worker, but around $90 \%$ had consulted their GP within the previous 12 months, on average six times each (Kendricket al, 1994). This is a relatively high rate compared with the average rate for adults of around three consultations per year. However, $10 \%$ had not been seen by their GPs for more than 12 months, a figure similar to other case register studies (Hassall \& Stilwell, 1977; Nazareth et al, 1993b).

Analysis of case note entries for the previous 12 months revealed the nature of long-term mentally disordered persons' contacts with their GPs. Overall, $76 \%$ had consulted for minor physical disorders, $12 \%$ for serious (potentially lifeshortening) physical disorders, $77 \%$ for repeat psychotropic prescriptions, and $48 \%$ for sickness certificates ( $66 \%$ of the men and $36 \%$ of the women). However, GP involvement in these persons' mental health problems was less frequent by comparison. Evidence of review of elements of the formal mental state examination within the preceding 12 months was found in only $32 \%$ of people, and GP-initiated changes in psychotropic drug regimens were recorded in only $20 \%$ of cases.

Psychotropic drugs may be prescribed for long periods in general practice in the absence of reviews of mental state and social functioning. In the 1970s general practice activity analysis data revealed that around half of all prescriptions of phenothiazines were repeat prescriptions where the person was not seen, and only one-third were given in follow-up consultations (Royal College of General Practitioners Birmingham Research Unit, 1978). However, it should be noted also that specialist psychiatric teams do not always review a person's treatment as often as they might. Holloway (1988) studied the drug regimes of 115 psychiatric day hospital patients and found that drug treatment was often prescribed for long periods with no evidence of a review of its effects or side-effects. Polypharmacy was common, with $72 \%$ of people on anticholinergic drugs, $69 \%$ on sedatives, $57 \%$ on antipsychotics and $32 \%$ on antidepressants. There was a high prevalence of tardive dyskinesia among the people with psychosis and Holloway considered that a trial of reduction in treatment was "long overdue" in many cases.

In our study (Kendricket al, 1994), while GPs were the most frequent medical contacts for people with schizophrenia, they did not seem to use the contact time to assess their mental and social problems. This finding is consistent with a number of other studies. Parkes et al (1962) found that most contacts with GPs in south London were brief consultations for repeat prescriptions, sickness certification and the treatment of physical problems. Boddington (1992) also found that more than $50 \%$ of the contacts 
between GPs and people with schizophrenia in Sheffield were limited to issuing repeat prescriptions of psychotropic drugs, and Nazareth et al (1993b) found that GPs in north London carried out significantly fewer 'disease-specific assessments' for people with schizophrenia than they did for people with chronic physical disorders including epilepsy, diabetes, rheumatoid arthritis and multiple sclerosis. They found that people with schizophrenia consulted more often with physical complaints than the average person, and suggested that this might divert doctors from reviewing important mental health issues.

These findings suggest that the care of people with schizophrenia and other long-term mental disorders might be improved by teaching GPs or practice nurses (or both) to carry out structured assessments.

\section{Structured patient reviews in general practice}

We conducted a randomised controlled trial teaching GPs to carry out structured assessments of long-term mentally disordered people in the 16 group practices in South Thames Region (Kendrick et al,1995). In eight practices GPs were given two teaching sessions on the problems of long-term mentally disordered people, and the use of a brief assessment (including mental state, social functioning and medication side-effects) designed for use in ordinary surgery appointments every six months for two years. The questions to be asked were included on an aide memoire placed in the person's general practice records (available from the author upon request). The impact on the process of care was measured by examining the person's general practice medical records.

Overall, 440 long-term mentally disordered people were identified in the 16 practices, of whom 253 (58\%) were suffering from chronic psychotic disorders. After two years, follow-up data were obtained on $85 \%$. Of 171 intervention group patients, $73 \%$ received at least one structured assessment but only $15 \%$ received four assessments. These assessments were associated with changes in the process of care of people in the intervention group. The proportion of patients whose psychotropic medication was changed increased from around $50 \%$ in the two years before intervention to $67 \%$ in the two years after, mainly due to changes in neuroleptic drug prescriptions. In addition, the proportions of patients referred for psychosocial problems increased, from around 15 to $20 \%$ for referrals to psychiatrists, and from around 5 to $15 \%$ for CPN referrals. There were no significant differences in measures of physical care, hospital admissions, self-harm, or use of the Mental Health Act.

\section{Special schizophrenia clinics in general practice}

Most of the GPs in our study, who were self-selected for their interest in mental health, considered that there was not enough time in routine consultations to carry out the structured assessments. An alternative approach has involved setting up special clinic sessions in practices to assess people with schizophrenia, similar to those for asthma and diabetes now widespread in general practice. Nazarethet al (1996) carried out a pilot study of such an approach in four London practices and found it to be generally feasible, although attendance dropped considerably at a second clinic session three months after initial assessment. The special session approach might require a change in funding, to pay for the additional space and the practice nurse's time. Special disease management payments for asthma and diabetes are already part of the GP contract with the health authority, and similar payments could, in principle, be made for a special approach to the care of schizophrenia. Currently, a trial of such an approach is being carried out from St George's in practices in Wandsworth, south London, involving payments of around $£ 80$ per person annually.

A third approach we have tried at St George's is to teach practice nurses who give depot neuroleptic injections (to at least four people each) to carry out brief structured assessments during injection appointments and to bring any problems found to their GP's attention. We found that the practice nurses were generally unsure and anxious about the problems of people with chronic psychosis, and keen to learn more about them. The effects of such assessments on the process of care and the outcome for patients are currently being examined in another randomised controlled trial.

It seems clear that educational initiatives aimed at increasing the quantity and quality of reviews of people with schizophrenia in general practice have the potential to change the process of care. General practice reviews of psychosocial care tend to generate referrals back to specialist care and subsequent changes in medication. However, this does not necessarily benefit the patient. Process measures are valid indicators of the quality of care 
only when they have been shown by research to lead to improved outcomes. There is little published evidence that referring people with schizophrenia back to specialist teams, or changing their drug treatment, is necessarily beneficial. It seems reasonable, however, to suppose that a review of their care is preferable to carrying on for years with the same treatment, risking neglect of problems or the development of irreversible side-effects.

\section{Physical problems}

Besides getting more involved in mental health care, GPs and practice nurses have a very important role to play in the physical care of people with schizophrenia. Rates of smoking exceed $50 \%$ among long-term mentally disordered people, compared with population rates of around $30 \%$. Obesity is common, in part due to the side-effects of psychotropic drugs. As a consequence, people with schizophrenia are at increased risk of developing chronic bronchitis, hypertension and angina (Kendrick, 1996). Mortality rates from cardiovascular and respiratory diseases are more than doubled in schizophrenia (Allebeck, 1989). GPs are usually aware of these risk factors, but do not intervene with long-term mentally disordered people to the same extent as with other people (Kendrick, 1993, 1996). Research is needed to establish whether health promotion, including dietary advice and nicotine replacement therapy, can succeed in people with schizophrenia. If not, then high mortality rates will persist, regardless of improvements in the care of a person's mental health problems.

\section{Improving the general practice care of schizophrenia}

As generalists, GPs have a prime responsibility to acquire and maintain competence across the breath of medicine, sufficient to make a meaningful initial response to any presentation whether medical, surgical, paediatric or psychiatric. Consequently, most GPs will not have a great depth of knowledge in any particular area. Few practice nurses have significant mental health nursing experience. For example, many GPs and virtually all practice nurses would not be able to describe akathisia, yet many neuroleptic drugs are prescribed by GPs and administered by practice nurses. Most GPs will have no idea about all the research that has been done on 'expressed emotion' and its impact on relapse rates, yet they are in a prime position as doctors to the whole family to give some simple advice about avoiding criticism and hostile remarks, or over-involvement and smothering of sufferers.

Therefore, the psychiatrist and CMHT may need to educate the GP and other primary care team members in the modern management of schizophrenia, including effects of medication, indications for treatment with newer antipsychotics, recognition and management of side-effects, psychosocial interventions with people and their families, and the application of mental health legislation.

Educational opportunities arise every time a person is referred by the GP, and every time a person is discharged into the community. The specialist's letter to the GP can be very educational, but needs to be brief enough to be read. Summaries of detailed out-patient assessments or hospital admissions which run to several pages, while undoubtedly useful as medical records, are often filed away quickly by the GP after scanning the contents looking for specific recommendations for management. It would be better to produce two records: a longer version for the hospital files, and a short one for the GP which outlines the reasons for management decisions, lists the effects and sideeffects of medication prescribed, and the indications and expected benefits of psychosocial interventions, perhaps referring to recent research.

Greater opportunities for mutual education arise where mental health professionals spend some of their time on general practice premises. However, the psychiatrist may need to take the initiative in arranging joint meetings of mental health and primary care teams, rather than simply settling for a shifted out-patient clinic model, which offers no increased contact or liaison compared with the traditional hospital clinic. Such meetings give both teams the chance to explore different perceptions of mental disorder and its management, allowing the development of agreed thresholds for referral and for discharge.

The psychiatrist and other community mental health professionals can help the primary care team to identify people with schizophrenia using the methods outlined above, and to draw up and maintain a practice schizophrenia register. The majority of practices should be able to produce a list of people on repeat prescriptions for antipsychotic drugs quickly from their computer. Such a tool is vital if all people with schizophrenia are to be remembered for possible reviews, especially the likely one-third who have been out of specialist contact for years. Where a person declines to be 
referred back to the specialist, the GP or practice nurse can review their care after discussion with the specialist team.

Many GPs and practice nurses will lack confidence in reviewing patients' care. They often do not know the best form of words to use to ask about hallucinations and delusions, for example, and may be afraid that by asking such questions they may open up a Pandora's box of problems to which they do not know how to respond and which might precipitate a crisis in a person's care. They need teaching about assessment and about the sources of help which can be mobilised for particular symptoms or social problems.

Psychiatrists already stretched by obligations to people currently in their care may be forgiven for baulking at the idea of going looking for more people with schizophrenia through the development of general practice registers. However, extra time spent in identifying persons out of touch with specialist services might save time in the long run, by picking up developing problems early, before they become severe enough to precipitate an urgent referral, perhaps requiring an expensive hospital admission that could have been prevented. In any case, many of those with schizophrenia who have no specialist contact are likely to be in stable remission and probably do not need referral if the GP can carry out a review of their care following discussion with the psychiatrist. Specialist teams that make an effort towards liaison and education are likely to benefit from the primary care team's increased confidence, which means that more routine care can be taken on in general practice. Written policies on shared care could be produced, perhaps based on recently developed consensus guidelines (Burns \& Kendrick, 1997).

\section{Guidelines for the primary care of schizophrenia}

In 1995 and 1996 a group of experts in general practice, psychiatry, nursing, and health economics met regularly at the Sainsbury Centre for Mental Health in London to produce brief consensus guidelines on the care of schizophrenia in general practice (Burns \& Kendrick, 1997). In 1998 these guidelines will be disseminated throughout the country.

The guidelines describe simple steps that practices can take to improve the care of their people with schizophrenia. However, such improvements will involve more work for both GPs and practice nurses, and will require an increase of expensive

\section{Box 2. What can be done to improve care?}

Teaching GPs to carry out structured assessments of long-term mentally ill people was associated with increases in treatment changes, especially of neuroleptics, and referrals back to secondary care services, especially to CPNs

Special clinics for schizophrenia care can be set up in general practice, involving the GP and the practice nurse

If improvements in the primary care of schizophrenia are to be generalised, then increased training is needed for primary care teams, and increased funding for the time and space needed to review patients systematically

resources such as time and space. The Department of Health should look urgently at the issue of providing special disease-management payments for schizophrenia, along the lines of those provided for asthma and diabetes clinics. Practices would of course have to demonstrate that they were providing good quality care, and this would best be done together with their local secondary care services. Hopes are high at this time of improving the primary care and shared care of people with schizophrenia (see Box 2).

\section{References}

Allebeck, P. (1989) Schizophrenia: a life-shortening disease. Schizophrenia Bulletin, 15, 81-89.

Boddington, J. (1992) Role of general practitioners in the care of long-term mentally ill patients (letter). British Journal of Psychiatry, 160, 568-569.

Burns, T. \& Kendrick, T. (1997) The primary care of patients with schizophrenia. A search for good practice. British Journal of General Practice, 47, 515-520.

Department of Health (1994) The Health of the Nation Key Area Handbook: Mental Illness (2nd edn). London: HMSO.

Hassall, C. \& Stilwell, J. A. (1977) Family doctor support for patients on a psychiatric case register. Journal of the Royal College of General Practioners, 27, 605-608.

Holloway, F. (1988) Prescribing for the long-term mentally ill. A study of treatment practices. British Journal of Psychiatry, 152, 511-515.

Jablensky, A. (1986) Epidemiology of schizophrenia: a European perspective. Schizophrenia Bulletin, 12, 52-73.

King, M. B. (1992) Management of patients with schizophrenia in general practice. British Journal of General Practioners, 42, 310-311.

Kendrick, T. (1993) Care of schizophrenia in general practice. British Journal of General Practioners, 43, 259-260.

- (1996) Cardiovascular and respiratory risk factors and symptoms among general practice patients with long-term mental illness. British Journal of Psychiatry, 169, 733-739. 
-, Sibbald, B., Burns, T., et al (1991) Role of general practitioners in care of long-term mentally ill patients. British Medical Journal, 302,508-510.

- Burns, T., Sibbald, B., et al (1994) Provision of care to general practice patients with disabling long-term mental illness: a survey in 16 practices. British Journal of General Practioners, 44, 301-305.

-, - \& Freeling, P. (1995) Randomised controlled trial of teaching general practitioners to carry out structured assessments of their long-term mentally ill patients. British Medical Journal, 311, 93-98.

Melzer, D., Hale,A. S., Malik, S. J., et al (1991) Community care for patients with schizophrenia one year after hospital discharge. British Medical Journal, 303, 1023-1026.

Nazareth, I., King, M., Haines, A., et al (1993a) Accuracy of diagnosis of psychosis on general practice computer system. British Medical Journal, 307, 32-34.

-, King, M., Haines, A., et al (1993b) Care of schizophrenia in general practice. British Medical Journal, 307, 910.

_- _ \& See Tai, S. (1996). Monitoring psychosis in general practice: a controlled trial. British Journal of Psychiatry, 169, $475-482$.

Parkes, C. M., Brown, G. W. \& Monck, E. M. (1962) The general practitioner and the schizophrenic patient. British Medical Journal, i, 972-976.

Royal College of General Practitioners Birmingham Research Unit (1978) Practice activity analysis 4: Psychotropic drugs. Journal of the Royal College of General Practioners, 28, 122-124.

Styles, W. McN. (1991) Training experience of doctors certificated for general practice in 1985-90. British Journal of General Practioners, 41, 488-491.

\section{Further reading}

Burns, T. (1996) Early detection of psychosis in primary care: initial treatment and crisis management. In The Prevention of Mental Illness in Primary Care (eds T. Kendrick, A. Tylee \& P. Freeling), pp. 246-262. Cambridge: Cambridge University Press.

- \& Kendrick, T. (1994) Schizophrenia. In Psychiatry and General Practice Today (eds. I. Pullen, G. Wilkinson, A. Wright, et al), pp. 194-208. London: Royal College of Psychiatrists and Royal College of General Practitioners.

Jenkins, R. \& Field, V. (eds) (1996) The Primary Care of Schizophrenia (2nd edn). London: HMSO.

\section{Multiple choice questions}

1. Studies of people with schizophrenia after discharge from hospital have shown:

a only around half attend psychiatric outpatients

b the majority of people are followed up by CPNs

c around $25 \%$ of people are looked after entirely in general practice

d most attend their GP's surgery for repeat prescriptions or sickness certificates

e the proportion followed-up by psychiatrists has greatly increased over the past 30 years or so.
2. Advantages of GP involvement in the care of people with schizophrenia include the following:

a for the majority of people GPs are their most frequent professional contacts

b most GPs have worked in psychiatric posts during their postgraduate training

c people who decline to attend psychiatric outpatients will often present themselves at their GP's surgery for care

$d$ most GPs have systems in place to deal with people who fail to attend for review

e a significant proportion of people receive their depot injections from practice nurses

3. GPs are likely to:

a have about 25-30 people with schizophrenia

b see people with schizophrenia frequently for physical problems

c review an individual's mental state regularly

$\mathrm{d}$ be willing to share the care of people with schizophrenia with the specialist team

e see $90 \%$ of people with schizophrenia in a 12month period.

4. General practitioners:

a consult on average for 15 minutes per person in surgery

b often give repeat prescriptions of antipsychotic drugs without seeing the person

c record the large majority of prescriptions for antipsychotics on computer

d should be aware which people are in receipt of the Care Programme Approach

e receive special payments for the care of people with schizophrenia.

5. A trial of teaching GPs to carry out structured assessments of long-term mentally disordered people resulted in:

a fewer referrals to mental health professionals

b more changes of patients' medication

c fewer admissions to hospital

$\mathrm{d}$ better physical care

e reduced self-harm.

\begin{tabular}{|c|c|c|c|c|}
\hline \multicolumn{5}{|c|}{ MCQ answers } \\
\hline 1 & 2 & 3 & 4 & 5 \\
\hline a $\mathrm{T}$ & a $\mathrm{T}$ & a $F$ & a F & a $F$ \\
\hline b F & b F & b $\mathrm{T}$ & b $\mathrm{T}$ & b $\mathrm{T}$ \\
\hline c $\mathrm{T}$ & c $\mathrm{T}$ & c $\mathrm{F}$ & c $\mathrm{T}$ & c $\mathrm{F}$ \\
\hline d $T$ & d F & d $\mathrm{T}$ & d $T$ & d F \\
\hline e $F$ & e $\mathrm{T}$ & e $T$ & e F & e $F$ \\
\hline
\end{tabular}

